\begin{tabular}{|c|c|}
\hline & $\begin{array}{l}\text { International Journal of Trend in Scientific } \\
\text { Research and Development (IJTSRD) }\end{array}$ \\
\hline 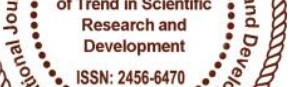 & International Open Access Journal \\
\hline 00 & ISSN No: 2456 - 6470 | www.ijtsrd.com | Volume - 2 | Issue -3 \\
\hline
\end{tabular}

\title{
Bionic Person for Issuing of RTO License
}

\author{
R. Preethi, P. Rajeshwari, R. Ananthi \\ UG Students, Department of Computer Science and Engineering, \\ Sri Muthukumaran Institute of Technology, Chennai, India
}

\begin{abstract}
This paper presents a novel approach for the computerization of driving license test system. In existing automated track has been implemented which includes in a driving test a candidate applying for license have to drive over all closed loop path in front of the authorities. He / She should be able to drive over the path without any support by resting his / her foot on the vehicle or land surface. If the candidate fails to do, he / she will be disqualified. The authorities will be watching the candidate manually. In proposed system, No authorities are needed for checking the candidates. Various sensors has been utilized for watching the candidate fails to their foot in the vehicle by differential output from the sensor can be monitored.The sensors installed are Load Sensor for checking the rest of foot on the vehicle or land surface. Smoke Sensor for checking the amount of smoke released while the accelerator raised. Grip Sensor for maintaining the gear speed. Gyroscope (Accelerometer Sensor) for checking whether the candidate drives the vehicle in correct direction or not. So, Bribes could not be encouraged for issuing the license and no illegal action will be involved. We can get RTO license easily without any problem.
\end{abstract}

\section{INTRODUCTION}

Presently, autonomous vehicles are a very hot topic in academia and industry. However, how to make autonomous vehicles practical is not only a technical problem but also includes safety, legal and social acceptance aspects among others. In recent days life technology has been developed and the growing technology introduces many advances in day today

life. This project helps to get driving license for the candidate without any biased interference of authorities. Conversely, advanced driver assistance systems that involve human interaction are more practical for applications. In anomaly detection in traffic scenes is implemented through spatial-aware motion reconstruction to reduce traffic accidents resulting from drivers, unawareness or blind spots. One person is dying road accident in every 30 seconds because of ineligible drivers with illegal license. So, it is very important to disassociate the driving ability test from the licensing authority. International Finance Corporation indicates that the process of obtaining driving license in India is a distorted bureaucratic one. The independent survey conducted shows that close to 60 percent of license holders did not even have to take the driving license test and 54 percent of them were untrained o drive.

\section{EXSISTING SYSTEM}

In present day, manual verification of an RTO officers is required in the testing field.RTO himself fixing his stare at the many number of test takers undertaking their license test. So, human error likes observation, favoritism and corruption. One or more inspectors from motor vehicle department has to stay long hours in the fields. Complicated process for getting the license, time consuming process we get a physical copy or smart card, every time the license paper / smart card need to carry, no any centralized database for verifying the license so for each state and internally for each district separate offices maintains the database. Bribes for getting license from authority in illegal manner are involved in the issuing of license. 
Automated track for automatically checking the candidate whether they move in correct direction or not.IR sensor monitor candidate's performance and it will be processed by another Arduino system connected to laptop or pc. The registered person entering for license test will be authenticated by using finger print sensor. So that they built automatically select or reject by the system. so, using this method, it may give incorrect readings. Partial automation of issue of driving license through lab view. A lab view system with sensors has been developed for watching the candidate. It was processed by micro controller and candidate is eligible or not decided. In this creates some problems are, Proximity sensor is used to identify the candidate leg positions alone, no other sensor is used to check the other driving position of the candidate. Candidate information is not available in other RTO office.

\section{SYSTEM OVERVIEW}

In automated track, only direction sensation can be done. Whether the candidates rest their foot on the land surface cannot be detected. No authorities are needed for checking the candidates fig[1]

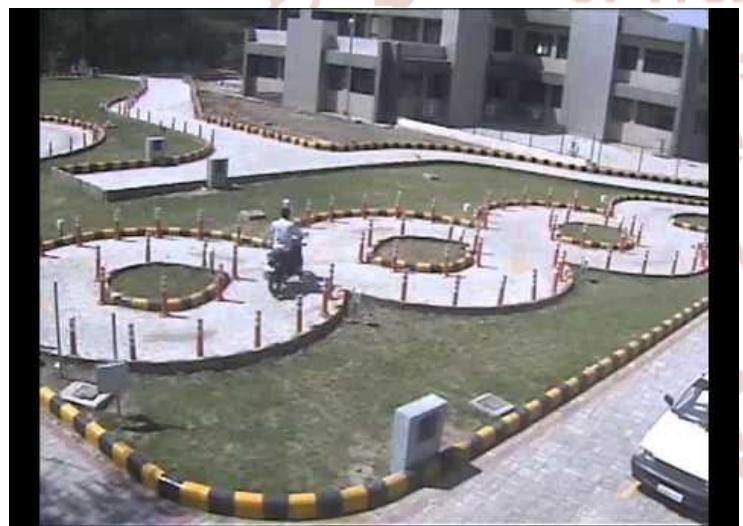

Fig [1]: Overview of Track for the candidate to drive their vehicle

The issuing of RTO license, many sensors are installed such that, Load sensor, gyroscope, smoke sensor, accelerometer sensor. Using this sensor, whether the candidates are eligible or not for issuing rto license. No authorities are involved for checking the candidate's performance. Gyroscope is used to check the candidates for direction. Accelerator is used to check the gear speed. Load sensor is used to checking the rest of foot on the vehicle or land surface. Those sensors are installed the driving test vehicle. One experience officer drive the testing vehicle and store all the values. Then the candidates are driving in the test vehicle and all the values are measured by sensors and comparing those sensors values and already stored values. Finally, after finish the driving test, candidates will show the result using LCD display. So, Time is reduced for waiting the result of license and no bribes are involved for issuing license. Accidents are reduced.

\section{ARCHITECTURE}

Arduino controller is used for read inputs from sensors and connects to the system. Smoke sensor, load sensor, grip sensor and gyroscope sensors are connected to the Arduino controller. We can connect 5v power supply and ground connection the Arduino controller. These values are reading from sensors and compare it original range values. UART convertor are connected from Arduino to computer / server. It checks the values and gives the result to LCD display for candidate's purpose and store the server for officers.

\section{Vehicle Unit}

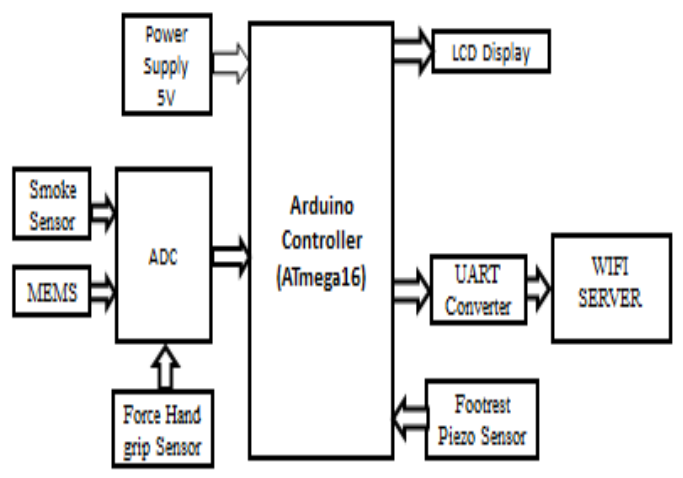

Fig [2] : Architecture of the system

Various sensors as shown in fig[2] have been utilized for watching the candidate whether they fail to rest their foot on vehicle by differential output from the sensor can be monitored. The sensors installed are Load sensor for checking the rest of foot on the vehicle or on the land surface. Smoke sensor for checking the amount of smoke released while the accelerator is raised. Grip sensor for maintaining the gear speed. Gyroscope (Accelerometer sensor) for checking whether the candidate drives the vehicle in correct direction or not.

Automation is the main domain of this architecture. The term automation inspired by the earlier word was established by an automation department of FORD. 
Automation can be defined as the technology by which process or procedure is performed without human assistance. Automated driving implies that you as a driver have given up the ability to drive (i.e.all appropriatemonitoring, agency and action functions) to the vehicle automation system. Automation has been achieved by various means including mechanical, electrical, electronic devices and computers usually in combination.

\section{PROPOSED DRIVING SYSTEM}

In automated track, only direction sensation can be done. Whether the candidate rests their foot on land surface cannot be detected. No authorities are needed for checking the candidates. Load sensor, Gyroscope, Gridsensor, Smoke sensor are installed to predict the accurate eligibility of the candidate.

\section{LICENSING SERVER ARCHITECTURE}

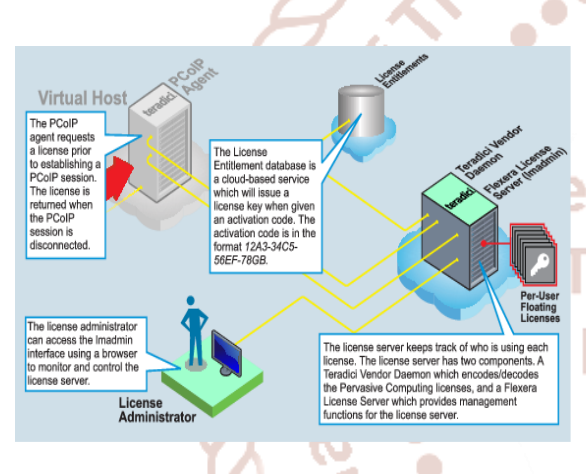

Fig [3]: Architecture of licensing server

The licensing server architecture fig[3] of the issue of RTO license involves the server, client and the database that is stored in the server about the candidate who appears for the driving test and the details of the candidates who pass / fail in the test are maintained by the licensing server .

\section{SYSTEM MODULES}

he system modules of the proposed RTO license issue include the following

$>$ Checking the amount of smoke.

$>$ Checking the gear speed.

$>$ Checking whether the candidate rests their foot on the vehicle or on the land surface

$>$ Checking the direction of the vehicle.

\section{a. ARDUINO CONTROLLER}

Arduino is an open source computer hardware and software company, project, and user community that designs and manufactures single board microcontrollers and microcontroller kits for building digital devices and interactive objects that can sense and control objects in the physical and digital world. The project's products are distributed as open-source hardware and software, which are licensed under the GNU Lesser General Public License (LGPL) or the GNU General Public License (GPL), permitting the manufacture of Arduino boards and software distribution by anyone. Arduino boards are available commercially in preassembled form, or as do-ityourself (DIY) kits.

Arduino board designs fig[4] use a variety of microprocessors and controllers. The boards are equipped with sets of digital and analog input/output (I/O) pins that may be interfaced to various expansion boards or Breadboards (shields) and other circuits. The Arduino project provides an integrated development environment (IDE) based on the Processing language project.

A minimal Arduino $\mathrm{C} / \mathrm{C}++$ program consist of only two functions:

- $\quad \operatorname{setup():This~function~is~called~once~when~a~sketch~}$ starts after power-up or reset. It is used to initialize variables, input and output pin modes, and other libraries needed in the sketch.

- $\operatorname{loop}($ ):After setup() has been called, function $\operatorname{loop}()$ is executed repeatedly in the main program. It controls the board until the board is powered off or is reset.

Most Arduino boards contain a light-emitting diode (LED) and a load resistor connected between pin 13 and ground, which is a convenient feature for many tests and program functions. A typical program for a beginning Arduino programmer blinks a LED repeatedly.

\#define LED_PIN 13// Pin number attached to LED. void $\operatorname{setup}()\{$

pinMode(LED_PIN, OUTPUT); //Configure pin 13 to be a digital output.

\} void loop() \{

digitalWrite(LED_PIN, HIGH); // Turn on the LED. delay(1000); // Wait 1 second (1000 milliseconds). digitalWrite(LED_PIN, LOW); // Turn off the LED. delay(1000); // Wait 1 second. 
Functions pinMode(), digitalWrite(), and delay(), which are provided by the internal libraries included in the IDE environment. The program is usually loaded in the Arduino by the manufacturer.

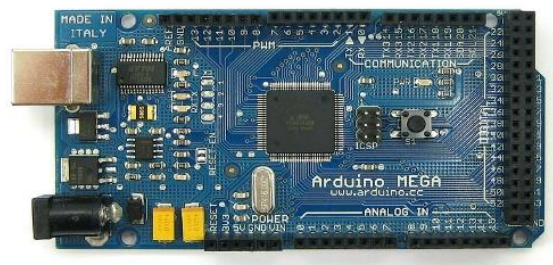

Fig [4]:Arduino Controller

The original Arduino hardware was produced by the Italian company Smart Projects. Some Arduinobranded boards have been designed by the American companies SparkFun Electronics and Adafruit Industries. As of 2016, 17 versions of the Arduino hardware have been commercially produced.

\section{a) SMOKE SENSOR}

The smoke sensor and the MQ-2 is a flammable gas fig[5] that detects the concentrations of combustible gas in the air and outputs its reading as an analog voltage. The sensor can measure concentrations of flammable gas of 300-10,000 ppm. The MQ-2 gas sensor is sensitive to LPG, i-butane, propane, methane, alcohol, hydrogen and smoke. They are used in gas leakage detecting equipments in family and industry and in portable gas detector.

- Supply voltage - 5V

- Sensitive to H2,LPG, CH4,SMOKE, ALCOHOL,PROPANE.

- Analog and Digital output

- Digital out is High or Low based on an adjustable present threshold.

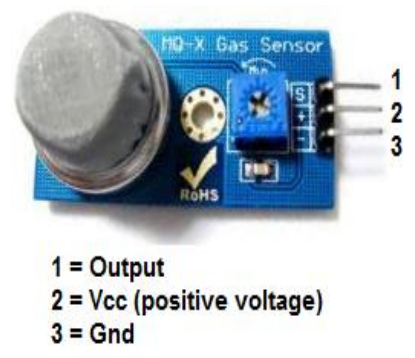

Fig [5] : Smoke Sensor

\section{b) ACCELEROMETER (GYROSCOPE)}

An accelerometer fig[6] is a device that measures proper acceleration. Proper acceleration being the acceleration (or rate of change of velocity) of a body in its own instantaneous rest frame. Accelerometers have multiple applications in industry and science. Highly sensitive accelerometers are components of inertial navigation systems for aircrafts and missiles. These are used to detect and monitor vibration in rotation machinery. Accelerometers are used in tablet computers and digital cameras so that images on screens are always displayed upright

Components used are:

- Piezoelectric

- Piezo sensitive

- Capacitive

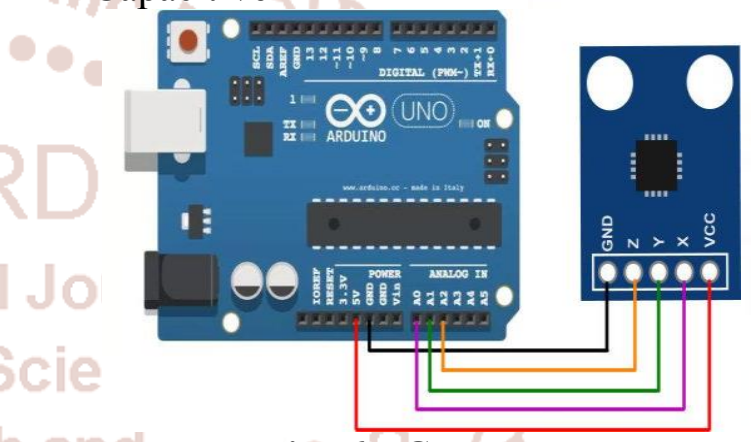

Fig [6]: Gyroscope

\section{c) PRESSURE SENSOR}

A Pressure sensor is a device for pressure measurement of gases or liquids. Pressure is an expression of the force required to stop a fluid from expanding and is usually stated in terms of force per unit area. A pressure sensor usually acts as a transducer; it generates a signal as a function of the pressure imposed. For the purposes of this article such a signal is electrical.

\section{Piezoelectric}

Uses the piezoelectric effect in certain materials such as quartz to measure the strain upon the sensing mechanism due to pressure. This technology is commonly employed for the measurement of highly dynamic pressures.

\section{Capacitive}

Uses a diaphragm and pressure cavity to create a variable capacitor to detect strain due to applied pressure, capacitance decreasing as pressure deforms the diaphragm. 


\section{d) UART CONVERTER}

A universal asynchronous

receivertransmitter (UART) fig[7] is a computer hardware device for asynchronous serial communication in which the data format and transmission speeds are configurable. The electric signaling levels and methods are handled by a driver circuit external to the UART. A UART is usually an individual (or part of an) integrated circuit (IC) used for serial communications over a computer or peripheral device serial port. UARTs are now commonly included in microcontrollers. A related device, the universal synchronous and asynchronous receiver-transmitter (USART) also synchronousos. electrically in parallel is the only way to increase the charge output.

\section{Shear effect}

The charges produced are strictly proportional to the applied forces and independent of the element size and shape. For elements mechanically in series and electrically in parallel.

\section{f) MEMS}

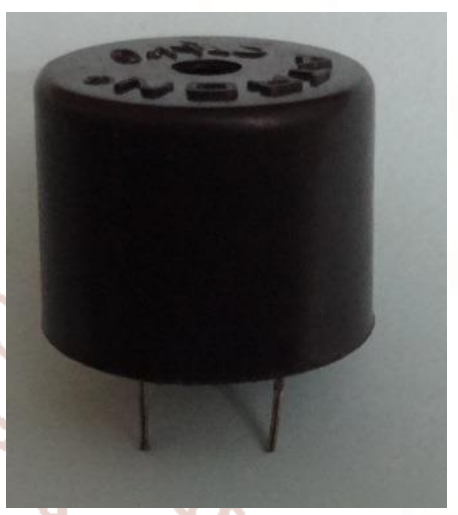

Fig $[8]$ : Piezo Sensor

Mems may refer to:

- Mem (computing), a measurement unit for the number of memory accesses used or needed Fig [7] : UART converter by a process, function, instruction set, algorithm or data structure

\section{e) PIEZO SENSOR (FOOTREST SENSOR)}

A piezoelectric sensor fig[ $[8]$ is a device that uses the piezoelectric effect, to measure changes, in pressure, acceleration, temperature, strain, or force by converting them to an electrical charge. The prefix piezo- is Greek for 'press' or 'squeeze'.

The way a piezoelectric material is cut produces three main operational modes:

- Transverse

- Longitudinal

- Shear.

\section{Transverse effect}

A force applied along a neutral axis (y) displaces charges along the $(\mathrm{x})$ direction, perpendicular to the line of force. The amount of charge depends on the geometrical dimensions of the respective piezoelectric

\section{Longitudinal effect}

The amount of charge displaced is strictly proportional to the applied force and independent of the piezoelectric element size and shape. Putting several elements mechanically in series and
- Microelectromechanical systems, the technology of very small devices

- Modular Engine Management System, an electronic control system used on engines in passenger cars built by Rover Group

Microelectromechanical system is the technology of microscopic devices, particularly those with moving parts. It merges at the nano-scale into nanoelectromechanical systems (NEMS) and nanotechnology.

MEMS are made up of components between 1 and 100 micrometres in size (i.e., 0.001 to $0.1 \mathrm{~mm}$ ), and MEMS devices generally range in size from 20 micrometres to a millimeter (i.e., 0.02 to $1.0 \mathrm{~mm}$ ), although components arranged in arrays (e.g., digital micromirror devices) can be more than $1000 \mathrm{~mm}^{2}$. They usually consist of a central unit that processes data (the microprocessor) and several components that interact with the surroundings such as micro sensors 


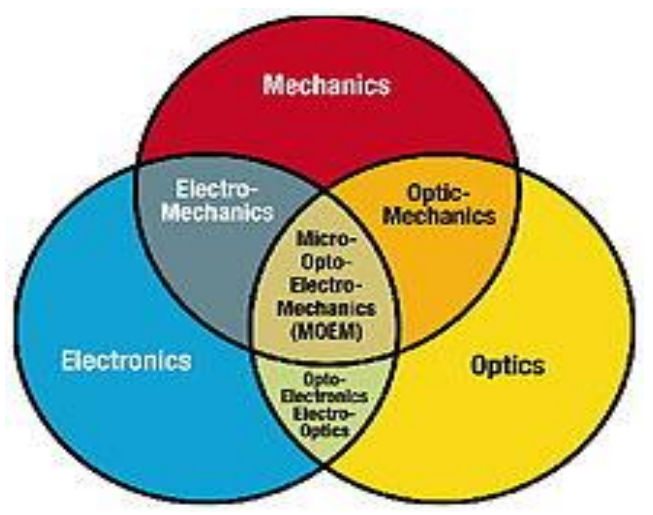

Fig[9] : MEMS representation

\section{g) GRIP SENSOR}

A grip sensor device and a grip sensing method are provided. The grip sensor device includes an antenna that is formed of metal within a mobile electronic device and communicates a signal of a first frequency and a grip sensor module that is formed within the mobile electronic device, is electrically connected to the antenna, and outputs a proximity detection signal according to proximity of an external object to the antenna.

\section{MERITS OF THE SYSTEM}

By using this, the candidate who fails to keep their foot in the vehicle can be monitored by sensor. To make the clear and efficient test of any driver by tracking the driving of a person while doing driving test.

Issue of license through illegal actions such as bribes can be overcome. With the automated testing machine, we can generate the real time result. if the driver result is pass or fail .it will display on android application. Based on number of errors made by the driver, the proposed system compares it with the threshold no of mistakes and decides whether to issue the license or not.

No authorities are needed for checking the candidates.

\section{DEMERITS OF THE SYSTEM}

Assumes a lot of technological presence in the environment it proposes a chip-based rule system to evaluate driver mistakes.

\section{CONCLUSION}

A successful implementation of predicting the driver behavior is implemented using variety of sensors. The propose system is designed for automatic driving license issuing using ATMega2560. This system monitors the person who applied for two wheeler license to find whether the candidate is eligible for getting the license.

The monitoring is performed for his/her driving position, foot control, throttle response and condition of vehicle etc, they will automatically select or reject by the system. It reduces the man power and there is no change for any illegal license. This system can be utilized for multiple applications for reducing the accidents and crime rate, maintain the database of candidates in RTO office, etc.

\section{FUTURE SCOPE}

Implementing this license automation can be extended to support global RTO by developing it with Internet of Things server (IOT). So that we globally access the data and results of the license test.

\section{REFERENCES}

1. "Automatic Two-Wheeler Driving License System by using Labview "D. Sarathkumar, C.K. Sathishkumar,S. Nithya,IJAREEIE, Vol.5, Issue 4, April 2016.

2. "Automatic Driving License Test with Android Application" Vanhere Payal Gopinath, Pagare Sneha Sunil, Aware Rupali Shantaram,Prof. Snehal S.Somwanshi,IJIRCCE, Vol.5, Issue 1, Jan2017.

3. "Automated Driving Test System "RashmiKonapanavar.Et.al. Int. Journal of Application,ISSN: 2248-9622,Vol.7, (Part - 2) July 2017 , pp.46-49.

4. "Automation of driving license test using wireless sensor network",Ms. Suvarna A. Dodke, p-ISSN: 2395-0072,Volume: 02 Issue: 08 Nov-2015.

5. "Design and implementation of a controller for Automated Driver Evaluation", Ashok Kumar, Neeharikha Gogineni and Swapna Gogineni 9781-4244-7042-6 / 10, 2010 IEEE.

6. P. Koopman and M. Wagner, "Autonomous vehicle safety: An interdisciplinarychallenge," IEEE Intell. Transp. Syst. Mag., vol. 9, no. 1, pp. 90-96, Jan. 2017.

7. Y. Yuan, D. Wang, and Q. Wang, "Anomaly detection in traffic scenes viaspatial-aware motion reconstruction," IEEE Trans. Intell. Transp. Syst., vol. 18, no. 5, pp. 1198-1209, Mar. 2017. 
8. B. Zhang et al., "A surround view camera solution for embedded systems,"in Proc. IEEE Conf. Comput. Vis. Pattern Recognit. Workshops, Jun. 2014, pp. 676-681.

9. M. Yu and G. Ma, " $360^{\circ}$ surround view system with parking guidance," Driver Assist. Syst., vol. 7, no. 1, pp. 19-24, 2014.

10. "360" wrap-around video imaging technology ready for integration with fujitsu graphics SoCs," Fujitsu Microelectron. America, Inc., Sunnyvale, CA, USA, Tech. Rep., Feb. 2011. [Online]. Available:

https://www.fujitsu.com/us/Images/360_OmniVie W_AppNote.pdf

11. M. Lin, G. Xu, X. Ren, and K. Xu, "Cylindrical panoramic image stitching method based on multicameras," in Proc. IEEE Int. Conf. Cyber Technol. Autom., Control, Intell. Syst., Jun. 2015, pp. 1091-1096.

12. Z. Hu, Y. Li, and $\mathrm{Y}$. Wu, "Radial distortion invariants and lens evaluation under a singleoptical-axis omnidirectional camera," Comput. Vis. Image Understand., vol. 126, no. 2, pp. 1127, 2014.

13. M. Schönbein, T. Strauß, and A. Geiger, "Calibrating and centering quasi-central catadioptric cameras," in Proc. Int. Conf. Robot. Autom. (ICRA), May 2014, pp. 4443-4450.

14. C. S. Fraser, "Automatic camera calibration in close range photogrammetry," Photogramm. Eng. Remote Sens., vol. 79, no. 4, pp. 381-388, 2013.

15. Z. Zhang, "A flexible new technique for camera calibration," IEEE Trans. Pattern Anal. Mach. Intell., vol. 22, no. 11, pp. 1330-1334, Nov. 2000.

16.Z. Zhang, "Camera calibration with onedimensional objects," IEEE Trans. Pattern Anal. Mach. Intell., vol. 26, no. 7, pp. 892-899, Jul. 2004.

17. Q. Wang, C. Zou, Y. Yuan, H. Lu, and P. Yan, "Image registration by normalized mapping," Neurocomputing, vol. 101, pp. 181-189, Feb. 2013.

18. H.-T. Chen, "Geometry-based camera calibration using five-point correspondences from a single image," IEEE Trans. Circuits Syst. Video Technol., to be published.
19. E. A. Bier and K. R. Sloan, "Two-part texture mappings," IEEE Compute Graph. Appl., vol. 6, no. 9, pp. 40-53, Sep. 1986.

20. P. J. Besl, "Geometric modelling and computer vision," Proc. IEEE, vol. 76, no. 8, pp. 936-958, Aug. 1988.

21. M. A. Ruzon and C. Tomasi, "Alpha estimation in natural images," in Proc. CVPR, vol. 1. 2000, pp. $18-25$.

22. A. Levin, D. Lischinski, and Y. Weiss, "A closedform solution to natural image matting," IEEE Trans. Pattern Anal. Mach. Intell., vol. 30, no. 2, pp. 228-242, Feb. 2008.

23. M. Salvi and K. Vaidyanathan, "Multi-layer alpha blending," in Proc. Meet. ACM SIGGRAPH Symp. Interact. 3D Graph. Games, 2014. 